\title{
Les Idées D'Avant-Garde Dans La Musique Bulgare Pour Piano Dans La Seconde Moitié Du XX ${ }^{\mathrm{ème}}$ Siècle*
}

\author{
Angela Tosheva
}

Le présent article ainalyse le chemin parcouru par la musique contemporaine d'avant-garde en Bulgarie vu par un interprète de musique pour piano.

La musique bulgare d'avant-garde pour piano reste à découvrir l'échelle internationale à cause des idées originales qu'elle comporte, pour leur temps; créée par des compositeurs bulgares parmi lesquels nous pouvrons distinguer Konstantine Iliev, Lazare Nikolov, Ivan Spassov, Vassil Kazandjiev et également Guéorgui Mintchev et Dimiter Hristov en tant que compositeurs aux idées particulièrement modernes.

L'isolament sévère à laquelle la Bulgarie fut soumise au sein de l'univers culturel mondial, comparé aux autres pays de régime communiste de l'Europe de l'Est explique le repliement interne dans lequel les compositeurs d'avant-garde ont créé leurs oeuvres. Cette dépendance directe des décisions politiques du parti communiste bulgare à laquelle nos créations musicales furent soumises a eu pour effet de nous couper de toutes sources d'information sur le développement de la musique au-délà du Rideau

* Tradução do búlgaro pela autora. Revisão da tradução francesa por Antoine Robert. 
de Fer qui ne soient pas sujettes à leur intromission. Dans une telle atmosphère de tension et de détente soumise aux créations artistiques soviétiques officielles en Bulgarie, il y eut des oeuvres uniques en leur genre autant par rapport à l'époque de leur création que par l'avant-gardisme de leur idée de composition. Bien entendu, celles-ci furent reçues dans la majorité des cas par une vague de réprobation de la part des compositeurs reconnus officiellement et fidèles à la politique du P.C.B., ainsi que publiquement démasqués de leur, soit disant, formalisme et décadence. D'autre part, une telle situation de division entre auteurs autorisés et interdits ne peut qu'être temporaire, car à un certain degré les traditionnalistes ont préssenti l'impossibilité de supporter eux-mêmes le fardeau des découvertes créatrices des avant-gardistes qui d'une manière involontaire, ont stimulé leurs recherches futures.

À partir de 1950 les compositeurs bulgares qui se sont dirigés dans la voie semi-légale de l'avant-gardisme sont Konstantine Iliev (1922-1986) et Lazare Nikolov (1924)'. Après avoir terminé ses études à l'Academie Musicale à Sofia, Iliev suit le cours de composition en 1946-47 d'Alois Haba à l'Academie Musicale de Prague. Lors de son retour, il s'immerge dans la recherche de sa propre individualité créatrice dans un climat d'ambiance noncréatrice créé par des collègues défiants. Cependant sa volonté de création réussit à dominer tout ceci et dès 1949 il crée son Concerto Grosso pour piano, percussion et instruments à corde, qui fut écrit avec une technique de composition tout à fait nouvelle, pratiquement inconnue dans l'esthétique avant-gardiste de l'époque dans les pays de l'Europe de L'Est, y compris la Pologne et la Yougoslavie. Lazare Nikolov définit cette oeuvre comme étant très créatrice et précurseur d'une nouvelle époque dans la musique bulgare. Dans ses mémoires, dont l'édition est prévue en 1997, Konstantine Iliev écrit sur ses principes de base qui le menèrent à la création de son style compositionnel polyphonique modalement amplifié: "Ces grands pas que nous faisions avec Lazare Nikolov provenaient de la facilité du terrain sur lequel cheminait notre pensée, car nous n'avions rien créé de neuf 
jusqu'en 1950, sinon que nous avions reconstitué plusieurs étapes, traversées par la musique durant les quatre à cinq dernières décennies... La question portant sur la polyphonie contemporaine se dressait devant nous comme une nécessité. J'étais absolument convaincu que la musique s'achemine vers une libération complète du style harmonique, vers une polyphonie absolue. Ce que j'entends par polyphonie absolue, c'est l'endroit vers lequel ma pensée créatrice se dirige toujours, même aujourd'hui: mener les voix en visant la marche logique de chacune d'entre elles sans tenir compte des formations verticales et de leur interdépendance fonctionnelle. Bien entendu, je ne dénie pas le recours aux sonorités d'accords, mais la formation de ces accords provient du développement logique de voix se mouvant lentement, et non de rélations fixées auparavant".

Faisons une comparaison momentanée des événements survenus dans la vie musicale en Pologne et en Bulgarie.

La Pologne est l'unique pays socialiste dans lequel l'activité des compositeurs d'avant-garde n'a pas été sujette à répréssion ni pendant cette période (1948-49), ni après les événements de Hongrie de 1956, ni plus tard, après les événements de Tchécoslovaquie en 1968. Durant leur visite en Bulgarie à l'automne de 1955 et au printemps de 1956 les compositeurs polonais Serotzky, Kotonsky, Dobrovolsky, ont rencontré Konstantine Iliev qui leur a présenté sa Deuxième Symphonie, et Lazare Nikolov, sa Première Symphonie et son Concerto pour violon et orchestre. Dans la situation confuse et de surprise créé par ce qui fut écouté, Iliev et Nikolov subissent l'accusation que ce n'est pas une manière d'écrire de la musique. Durant l'automne de la même année (1956), au Premier festival Automne de Varsovie, les compositeurs polonais comprennent que la musique tonale n'a plus d'issue d'une façon genérale, et constituent leur lobby d'avant-garde en Europe, plus précisement après la composition Musica Zalobna par W. Lutoslawsky (1958). D'après Lazare Nikolov ce qui était consideré en tant que musique d'avant-garde pendant les années 50 en Italie, en Allemagne, en France et aux 
États Unis, n'a pas eu d'éxistence au-délà du Rideau de Fer, ni en Pologne pas plus qu'en Yougoslavie, à l'exception des oeuvres de Konstantine Iliev. Il y a bien entendu, peu d'importance de savoir qui d'entre ces compositeurs a été le premier à écrire une véritable oeuvre d'avant-garde, sinon que c'est un jeune compositeur tout à fait inconnu qui, sans disposer d'informations - des partitions, des stations radio découvertes au hasard ou bien des rencontres - a créé son propre langage de composition par lequel il a exprimé son point de vue sur la manière dont la musique devrait se developper aujourd'hui.

Nous trouvons une particularité post-romantique caractéristique de la musique pour piano écrite par K. Iliev et L. Nikolov dans les années qui suivent 1950: les principes classiques d'organisation de la forme sont employés en tant que moyen d'exposition des nouvelles idées d'avant-garde.

L. Nikolov est un des compositeurs de base de la musique pour piano seul. Il est l'auteur de sept sonates pour piano et d'une pièce de concert Reflets pianistiques, qui représentent une autobiographie sui generis reflétant des étapes créatrices traversées depuis 1950 jusqu'à 1990. Le langage musical qui en ressort devient universel pour l'auteur et s'intègre à tout niveau de procédé dans le contenu et la forme de ses oeuvres pour piano. L. Nikolov s'appuie sur la tradition typique de la sonate et la symphonie en quatre parties avec un Allegro de forme sonate. Toutefois toute la thématique, et le respect des structures de composition classique disparaissent lorsque la musique commence à se développer, bien que le schéma classique soit préservé. La structure de l'oeuvre se fait sentir par son développement dramaturgique de type sonate-symphonie qui est irrévocable pour Nikolov. Ceci est le résultat de l'insertion d'une note dramatique innée dans chacune des oeuvres de l'auteur.

Il y a une atmosphère dans l'espace musicale qui rappelle un mozaique, ce qui amène l'installation du matériel sonore d'une façon complémentaire dans une conséquence horizontale. 


\section{Exemple 1}

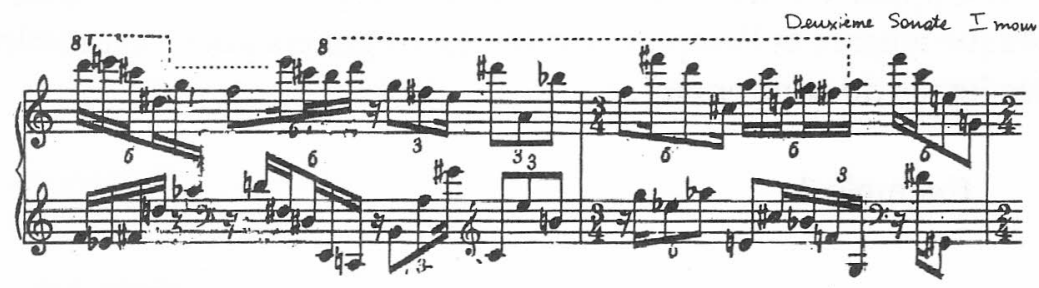

Les dialogues et les rélations entre les voix achèvent de développer un motif déjà exposé en un autre motif voisin, situé soit plus haut soit plus bas. Nous avons pour résultat une ligne complète qui se déroule en un mouvement constant, se meut, se renouvelle suivant la logique momentanée du compositeur. L'impréssion qui se crée est celle d'un mouvement musical libre, éloigné de toute limite possible. Cette ligne stylistique, chez Lazare Nikolov, est proche de l'idée de la forme momentanée de Stockhausen, mais nous avons ici une solution spécifique. Ce qui donne raison à la comparaison de ce type est le résultat qui est obtenu à partir d'une forme ouverte, ainsi que son édification qui se réalise sur le principe d'une métamorphose musicale incessante. La première oeuvre pour piano de L. Nikolov, est basée sur les mécanismes du système série mais de forme classique; la Seconde Sonate fut écrite en 1951.

\section{Exemple 2}

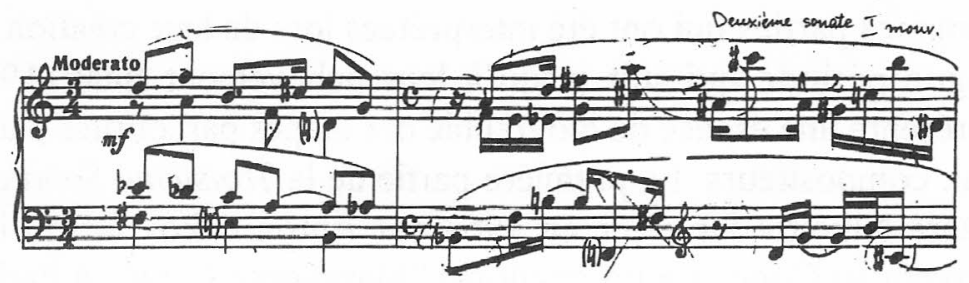

Il y a trois traits particulièrement caractéristiques dans la Seconde Sonate: le développement concentré qui frise le 
dépouillement, les thèmes initiaux en une texture d'accords se développant en série, ainsi que des. changements rythmiques à chaque mesure et l'emploi de rythmes irréguliers pour l'expression des lignes de contrepoint.

\section{Exemple 3}

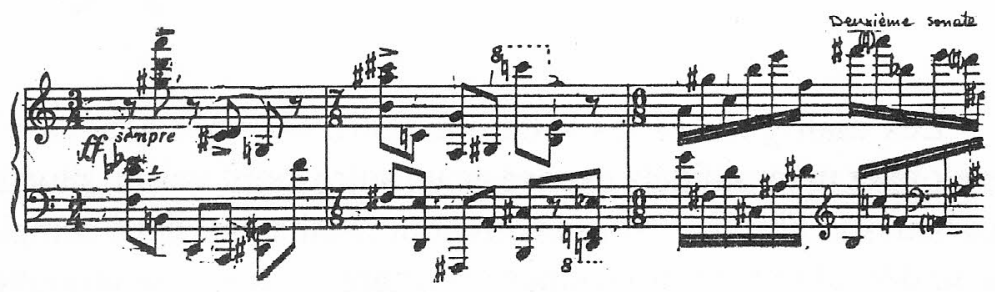

Nous sommes en présence d'une manière subconsciente de bulgarisation d'une écriture purement polyphonique. Nous rencontrerons une technique similaire dans la Toccata de Konstantine Iliev et dans ses Cinq pièces pour pianistes avancés.

L'évolution du langage de composition déjà décrit chez Lazare Nikolov se poursuit dans les sonates pour piano qui suivent, écrites après 1955. L'auteur lui-même déclare que c'est à partir de la Troisième Sonate (1955-56) et de son Quintette pour Piano et Cordes (1958) qu'il découvre son style personnel de composition. La Troisième Sonate de Nikolov a connu un destin très proche de celui de la Troisième Sonate de Pierre Boulez. Toutes deux ont été commencées la même année (1955), et ce ne sont que leurs premières parties qui ont été interprétées lors de leur création. La longue période qui suit jusqu'à leur achèvement final (1958) représente une espèce de biographie des étapes parcourues par les deux compositeurs. La première partie de la Troisième Sonate de Lazare Nikolov fut créée en 1963 par Anton Dikov lorsqu'il se présenta au Concours International "Marguerite Long" à Paris.

La Troisième Sonate de Lazare Nikolov représente la première véritable oeuvre d'avant-garde pour piano en Bulgarie. Le processus dramatique du parcours musical de la technique sérielle s'aprofondit 
chez Nikolov à partir de sa Quatrième Sonate (1964) où il commence à exploiter les possibilités de timbre du piano. L'atmosphère intensément dramatique de sa Sixième Sonate (1982) a été atteinte d'une façon magistrale à travers l'utilisation de toute la tessiture du piano. Cette Sonate, dediée à Konstantine Iliev, fait appel aux techniques pianistiques les plus diverses: ce sont les variantes de plusieurs accords initiaux qui se décomposent graduellement et se rassemblent en un mouvement de choral qui apparaît avec tous les moments-clef de ce cycle bipartite.

\section{Exemple 4}

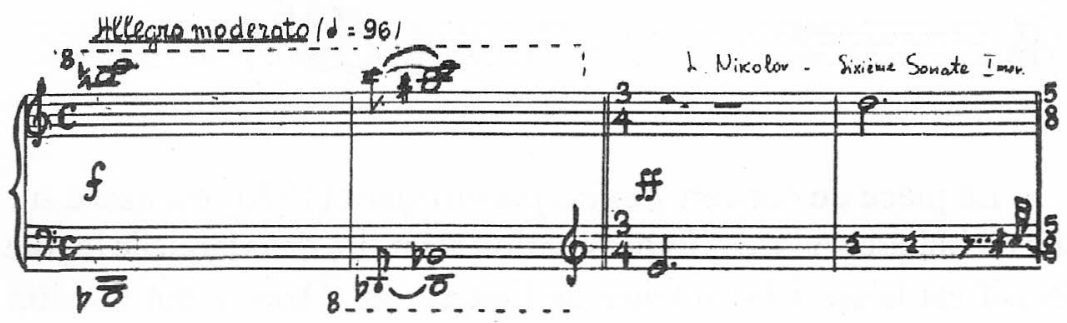

Exemple 5

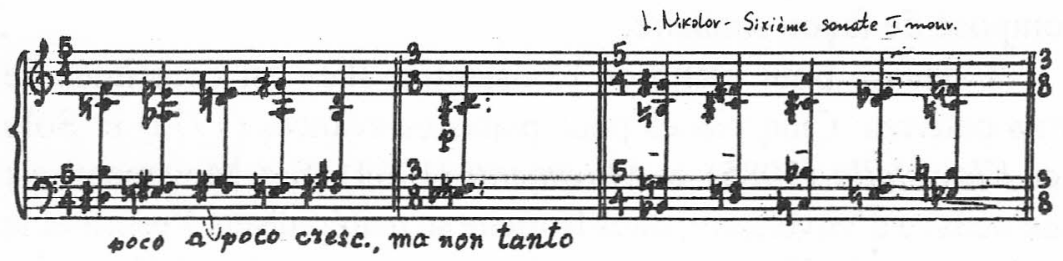

La dynamique puissante des chorals et leur résonnance modale nous rappellent l'atmosphère de Messiaen.

En 1991 Nikolov termine sa Septième Sonate, laquelle représente une synthèse de son oeuvre toute entière. La complexité et la perception difficile du public non-averti paraîssent diminuer: la technique complèxe de composition est soumise de façon très vive à une ligne découlant très impetueusement lorsque 
simultanément cette construction apparamment classique se modifie de façon complète jusqu'à la fin. La capacité notable de Nikolov réside en sa possibilité de généralisation tout de suite, avant et durant les moments finaux, alors que se crée une impréssion de séparation de la realité:

\section{Exemple 6}

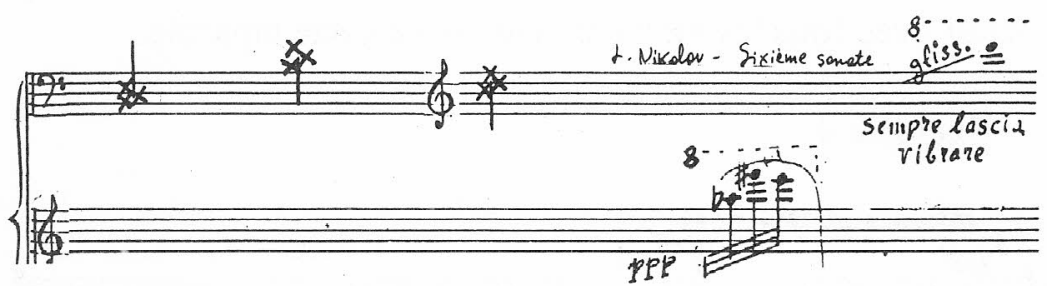

La pièce de concert Reflets pianistiques (1970) est basée sur une technique sonore d'improvisation aléatoire dans laquelle le rôle décisif est laissé à la fantaisie de l'interprète. Dans le but d'éviter les attaques de la critique, Nikolov donne le jour à cette oeuvre peu après que Vassil Kkazandjiev (1934) ait publié en 1975 son oeuvre dédiée au piano solo appelée Triomphe des cloches, qui fut composé de façon similaire.

L'oeuvre pour piano de Konstantine Iliev est constituée de trois oeuvres: Cinq pièces pour pianistes avancés (1972) et Solo per Klavistella (1985) et Movimenti (1964). Ses Movimenti est une oeuvre d'envergure, dans laquelle se matérialisent l'esprit et le credo musical d'Iliev en tant qu'avangardiste inébranlable, qui a recours aux principes de composition les plus stricts. Solo per Klavistella est dédiée à la célèbre pianiste bulgare Stella Dimitrova.

Movimenti fut écrite après le Premier festival de Musique Contemporaine Automne de Varsovie (1956). L'esprit créateur récemment découvert se fait sentir d'une façon très claire, démontrant combien l'auteur le domine de façon complète et comment il continue à le développer à travers ses nouvelles oeuvres, surtout ayant déjà terminé sa Cinquième Symphonie (1958). La 
Maîtrise d'Iliev se fait sentir au moment où il se sert de registres strictement polyphoniques, et même de moyens musicaux réduits, inspirés par l'habilité des flamands du XV-ème siècle.

Movimenti est composée de quatre parts: Movimento libero,

Exemple 7

Movimento variado,

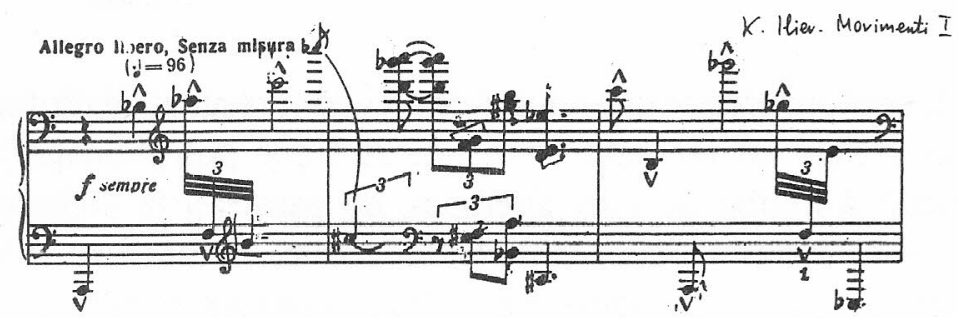

Exemple 8

Movimento iterrotto,

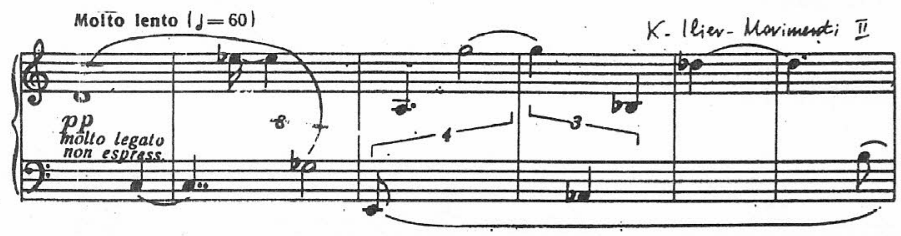

Exemple 9

Movimento vigoroso:

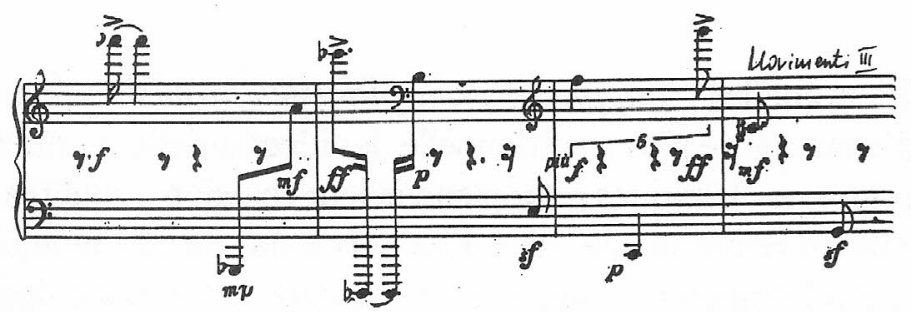


Exemple 10

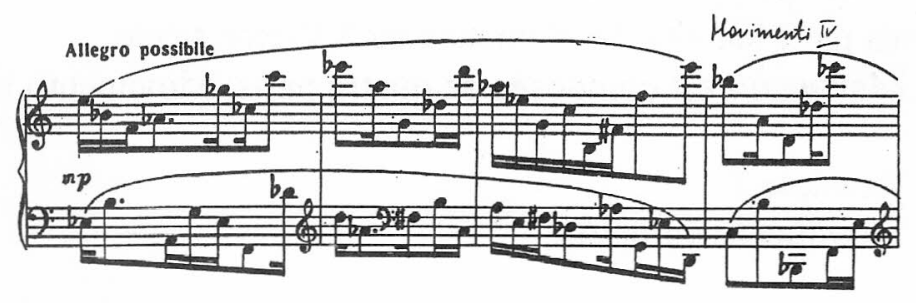

L'écriture précise que nous avons ici est le résultat de profonds calculs sériels sur une texture menée de façon polyphonique qui s'enrichit à l'infini de voix alternées, de pauses à la Webern et d'autres éléments sériels. Dans chaque partie, en fonction de leur caractère, il existe une figure qui se développe tout en ayant le son authentique du à sa construction modale amplifiée (En admettant que la modalité amplifiée représente l'archétype de la technique dodécaphonique). La dernière oeuvre pour piano de K. Iliev, Solo per Klavistella, est entièrement édifiée sur des figures modales caractéristiques.

Exemple 11

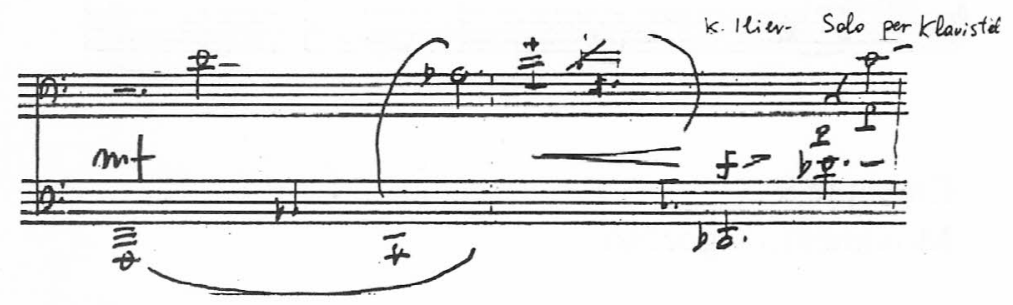

Bien que les Cinq pièces pour pianistes avancés soient d'un type d'avantgarde plus traditionnelle, les idées qu'elles contiennent - les principes classiques pour piano et leur éclosion - sont réalisées d'une façon remarquable. Iliev a recours à des formes typiques du piano: étude, nocturne, valse, toccata, dans le contexte desquels nous retrouvons en cherchant bien l'esprit de Chopin, Bartók, et 
Debussy, mais filtrés à travers le prisme d'un avantgardiste qui pose des questions et trouve les réponses.

La première pièce, intitulée Dédicace, est particulierement allégorique par le fait qu'un mouvement extrêmement élaboré, contenant une ligne de série typique, se développe sur un accord toujours ostinato de Do majeur à la main droîte. Le rythme et la hauteur sont sans égard pour les limitations. L'auteur donne libre cours à sa question de fond: est-ce que, aujourd'hui, le compositeur peut rester à l'écart des transformations révolutionnaires dans la pensée musicale intervenues après les années $50 \mathrm{du} X \mathrm{X}$-ème siècle et comment aurait-il vécu si celles-ci faisaient réellement partie de ses convictions créatrices.

\section{Exemple 12}

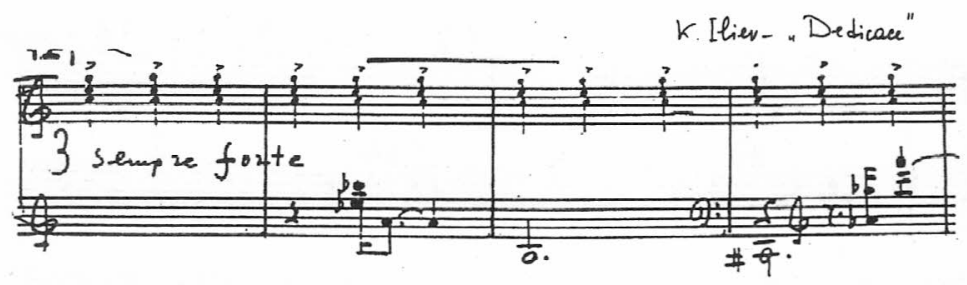

Exemple 13

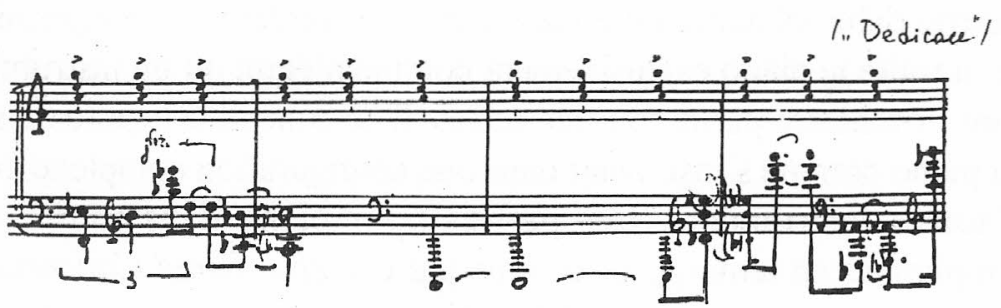

Dans sa seconde pièce, Étude, Iliev construit une ligne modale de quatre double croches, qui se transmet en un tempo rapide entre main gauche et main droîte. 
Exemple 14

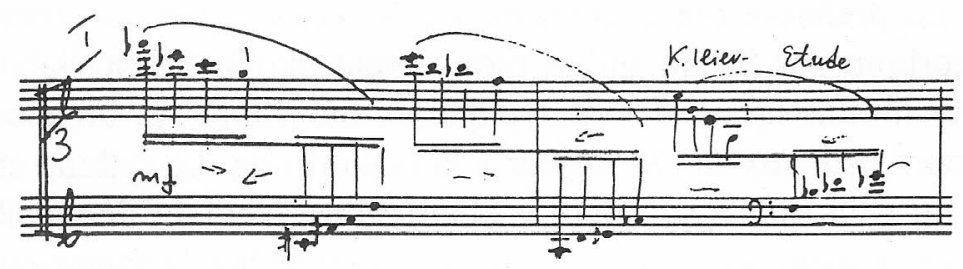

Nocturno recourt à une basse obstinée en un registre grave, sur lequel s'édifie une ligne éloignée dans le registre aigu, ce qui donne un effet d'éloignement abstrait. Cette oeuvre n'est pas sans évoquer le sens mélodique de Chopin.

Exemple 15

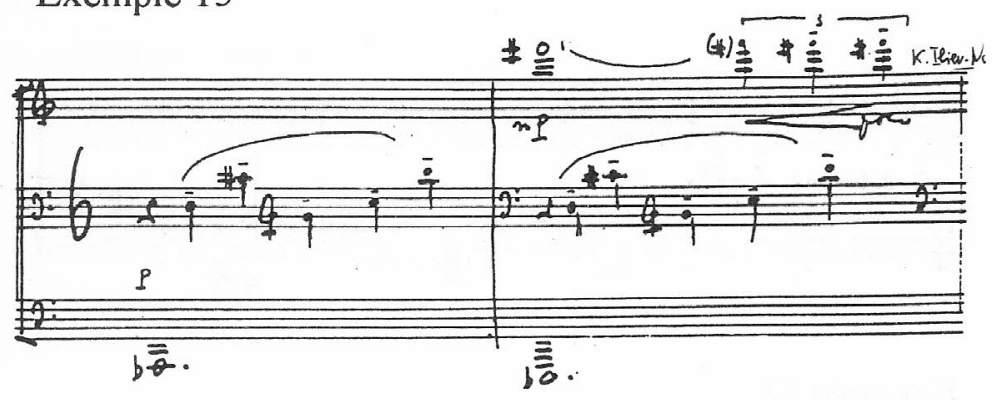

K. Iliev nous laissse un héritage d'oeuvres pour piano qui n'est pas très riche, dû au fait qu'en tant que chefd'orchestre et symphoniste né, il traite le piano exclusivement comme n'étant qu'un instrument dans l'orchestre parmi tous les autres. Il ne conçoit la beauté du son du piano comme s'inscrivant dans une configuration complexe avec d'autres instruments. Les parties pour piano dans toutes ses symphonies en témoignent comme par exemple Tempi Concertanti II pour clavecin et flute (1970). Nous retrouvons chaque fois la capacité d'Iliev d'écrire de façon diverse pour chaque partie instrumentale séparée en fonction de ses possibilités et spécificités.

Grand nombre de compositeurs de la génération suivante ont recours au langage contemporain pour l'écriture de leurs 
compositions, surtout à partir de 1965 jusqu'au printemps de 1968 lors du grand mouvement qui surgit dans le bloc des pays de l'Est et la pénétration de concepts tout à fait nouveaux et différents dans la vie musicale locale.

À cette époque, il y a de nouveaux noms qui apparaissent et s'affirmemt: Vassil Kazandjiev (1934), Ivan Spassov (1934), Tryphon Sylianovski (1923), Guéorgui Toutev (1935-1995), Guéorgui Mintchev (1938), Dimiter Hristov (1933). Ils témoignent de leur participation dans les procédés d'avant-garde qui pénétrent en Bulgarie de façon de plus en plus facile, à divers degrés ainsi qu'à des moments différents, se transformant en un champ fertile aux expériences individuelles. À cette époque, lorsque la technique sérielle, aléatoire, la combinaison de techniques néoclassiques, postromantiques et dodécaphoniques deviennent modernes en Bulgarie, la grande question qui se pose aux compositeurs concerne la place que devrait occuper et l'importance que devrait avoir le folklore. Nous pourrions à un certain degré expliquer la nuance nationaliste de cette question dans l'ambiance d'une démarche toujours plus dure de sauvegarde des frontières sonores du pays en présence des possibilités d'information de plus en plus diverses. C'est un fait réel qu'un grand nombre de compositeurs traditionnalistes et de critiques musicaux d'après 1965 utilisent le folklore en tant que masque pour pouvoir dénigrer chaque oeuvre atypique et plus nouvelle: l'on organise des commentaires publics, des auditions au travers desquelles, nonobstant, commencent graduellement à se former une attitude plus précise à l'égard des valeurs traditionnelles et de la provocation de la contemporaneité. Chaque compositeur d'avant-garde s'engage à sa façon à résoudre la question de la place que devrait occuper le folklore, trait typique de la culture musicale d'une école nationale renaissante de l'Europe de l'Est. Dans le cas de Lazare Nikolov, ceci représente sa variante idéalisée, son esprit qui se fait sentir à travers le contexte d'une oeuvre purement sérielle. En ce qui se rapporte à Konstantine Iliev, ceci est une juxtaposition de collage de deux textures apparamment incompatibles: la polyphonie sérielle et le folklore, aux rythmes irréguliers: 
136 Revista Música, São Paulo, v.7, n.1/2: 123-148 maio/nov. 1996

Exemple 16

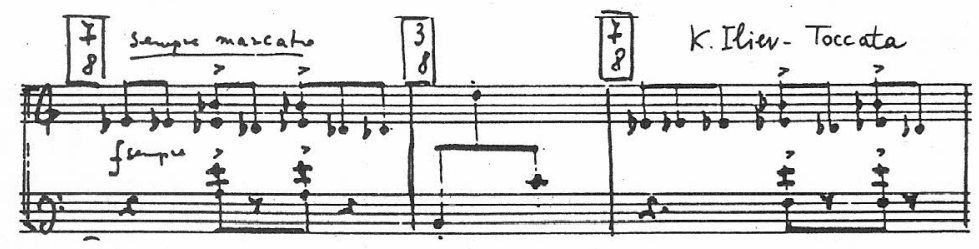

Pour Vassil Kazandjiev, ceci est un retour aux anciennes images bulgares et la genèse du paganisme avec le christianisme: des Koukeres $^{2}$ avec des icônes sur la base des méthodes aléatoires et sonores.

Exemple 17

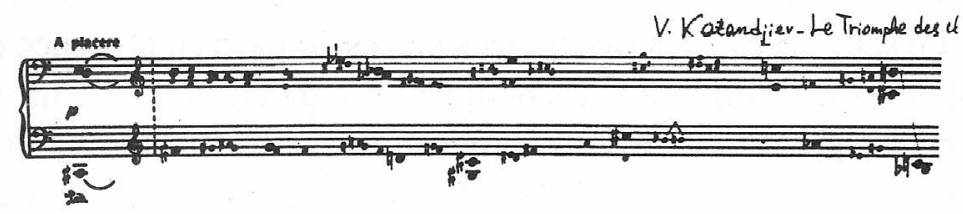

Exemple 18

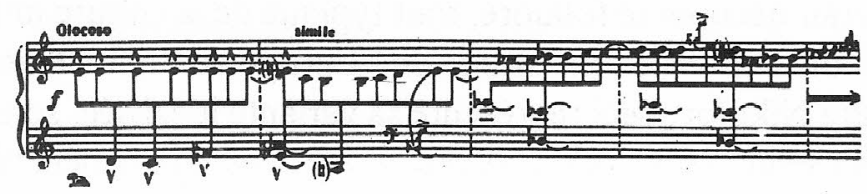


Pour Ivan Spassov, le folklore représente un mode de vivre et de penser, surtout dans ses oeuvres vocales, tandis que dans ses oeuvres pour piano il a recours à une technique sérielle exclusivement stricte, edifiée à la façon d'un maître, pour pouvoir exprimer des états d'âme émotionnels éphémères.

\section{Exemple 19}

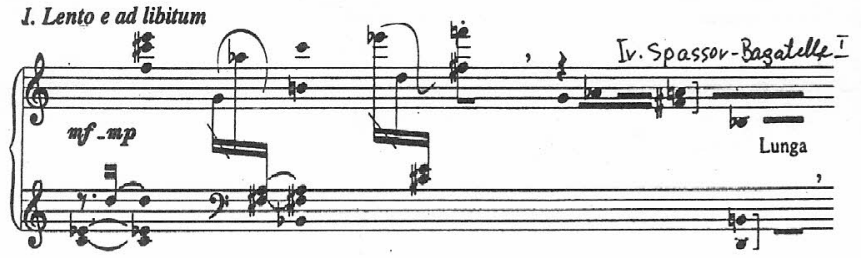

Pour Guéorgui Mintchev, le folklore représente une monumentale explosion d'énergie archétype sonore longtemps accumulée, qu'il atteint à l'aide d'improvisations sur des motifsrépliques folkloriques librement exprimés, par lesquels il crée une structure polydimensionelle de l'espace sonore et un jeu sonore savant sur des plans proches et éloignés.

Exemple 20

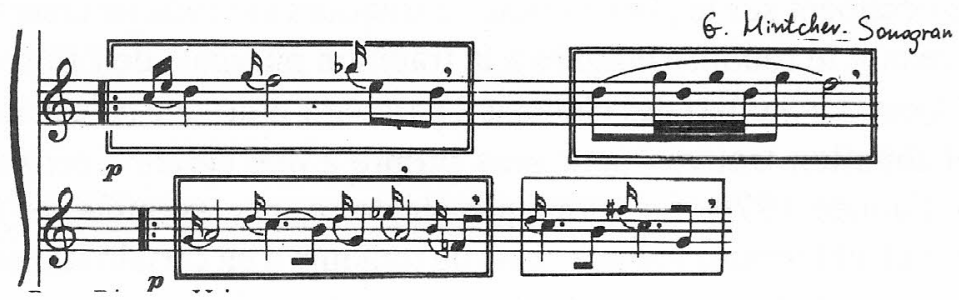


Pour Dimiter Hristov, le folklore est présent dans toutes ses oeuvres pour piano par l'emploi de microstructures antithétiques variantes, provenant pour la plupart de la tradition Shoppe (région avoisinant Sofia) de chant populaire.

Exemple 21

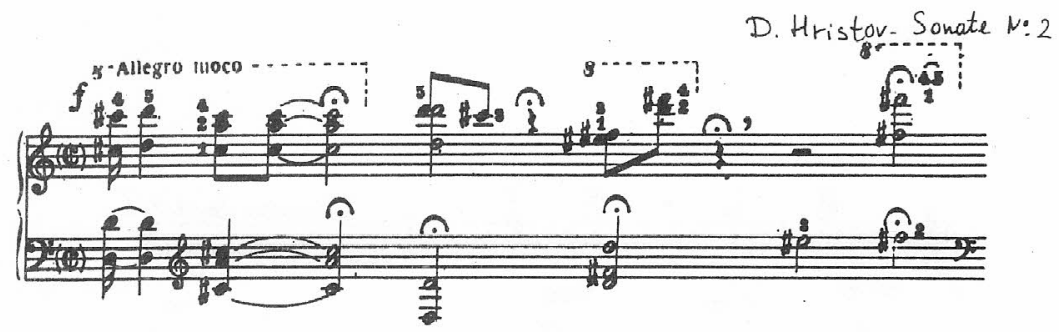

Exemple 22

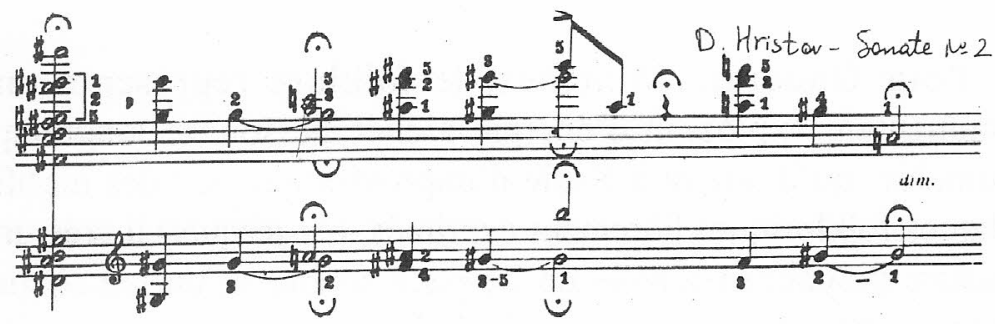

Une idée d'importance primordiale pour les compositeurs bulgares, qui ne dépend point de leur considération de la place qu'ils occupent sur le plan mondial, a toujours été celle de créer un pont reliant le folklore bulgare à la tradition musicale de l'Europe de l'Ouest. La plupart des chemins à parcourir pour une telle liaison et cohabitation trouvent leur sens propre à une certaine période, après l'année 1970. La technique aléatoire, par exemple, et les citations folkloriques mènent presque toujours à un certain statisme et un manque de diversité de la forme, mais celles-ci se trouvent plutôt dans la sphère des expériences naturelles de création sans conséquences à l'échelle internationale. 
Vassil Kazandjiev, en tant que représentant de la génération suivante de compositeurs, crée une oeuvre pour piano sans équivalent jusqu'alors (Le triomphe des cloches, 1975).L'auteur donne à l'interprète un rôle précis tout à fait nouveau d'improvisateur-co-auteur, pouvant mettre dans l'oeuvre toutes les ressources sonores du piano ainsi que sa vision individuelle sur la construction de la forme dans le temps. La libre alternance de 20 modèles, entre lesquelles seuls le premier et le dernier ont un endroit fixé, est plein de détails qui éxigent une élaboration précise, vu le rôle important de l'interprète et de son intuition envers la création de formes. Le son des cloches à tous les niveaux sonores, timbres et dynamiques possibles scellent de façon naturelle l'oeuvre complète écrite en un seul mouvement. Mais le plus interessant ne sont pas les sons: il y a des moments auxquels le son est completement déchargé de tout sens possible; il ne fait qu'exister tout simplement, dynamique ou statique, en un plan proche ou éloigné, dans un grondement ou dans un évanouissement continu de la sonorité. La séparation du son de sa caractéristique sémantique et verbale a été atteinte de façon exceptionnellement moderne, précisement dans une matière sonore littérale aussi proche de l'ouie humaine que celle des cloches. L'oeuvre, intitulée Le triomphe des cloches, de par son dépouillement et son économie de moyens, est l'une des plus réussies parmi les oeuvres bulgares contemporaines pour piano.

\section{Exemple 23}

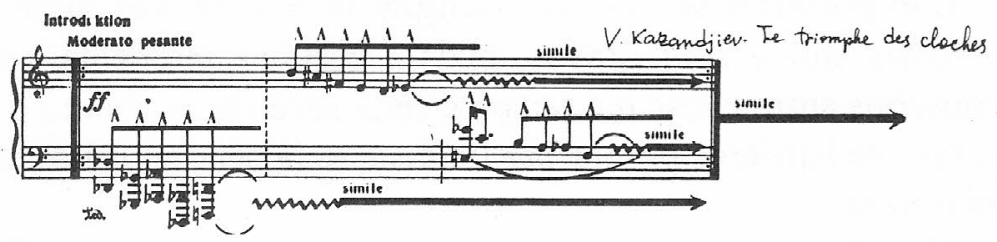


Ivan Spassov est le créateur d'un style de piano très personnel. Il est l'un des compositeurs bulgares les plus notoires par son don de façonner des chants folkloriques. La ligne particulière sur laquelle il s'engage dans la musique pour piano seul lui donne de plein droit une place parmi les compositeurs d'avant-garde la plus avancée du pays aussi bien qu'en Europe. D'après l'auteur lui même, chaque période historique et par conséquent chaque style ont leur propre langage qui reflète sans équivoque les tendances de leur époque. Aux dires d'Ivan Spassov, l'écriture naturelle du piano, durant la seconde moitié du XX-ème siècle, c'est la technique sérielle. Il est l'auteur du cycle L'Art de la Série représentant une réplique à Bach dans le contexte d'aujourd'hui (1978), 24 études pour piano en tant que réplique aux études de Chopin, la Première Sonate (1985), la Seconde et la Troisième, construites comme un seul cycle (1987), 24 bagatelles (1995) et une Pièce de style folklorique (1995). Toutes les oeuvres de cet auteur ont un aspect unique, mais ce qui les relie, c'est exactement l'absence de toute note inutile, l'unité d'émotion et de l'écriture.

\section{Exemple 24}

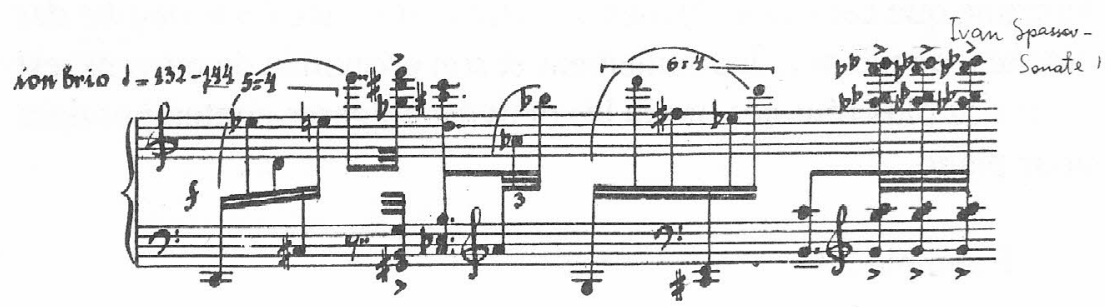

L'expression laconique souligne la beauté des instants s'envolant qui y sont graves, mais à certains moments nous découvrons autre chose qui tempère cette sévérité, et aboutit à ce résultat: les barrières posées par l'auteur se brisent pour l'espace d'un instant.

Une de ses dernières oeuvres pour piano, les 24 Bagatelles, est l'exemple de l'équilibre atteint entre rationnel et irrationnel, logique et émotion, réalisation technique et contenu. 


\section{Exemple 25}

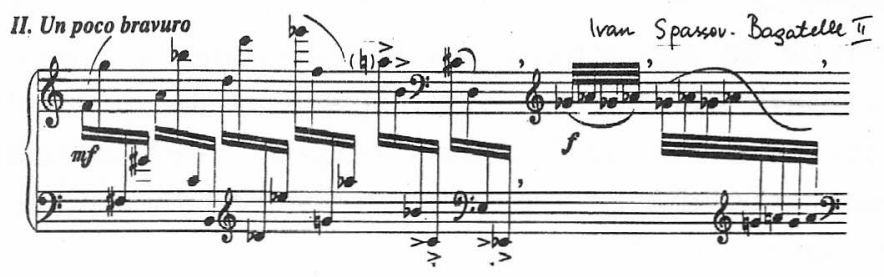

Les oeuvres sont très pianistiques dans leur ensemble, cependant elles exigent une préparation prolongée et attentive. La Pièce de stylo folklorique est la première oeuvre fournissant la preuve qu'Ivan Spassov relève le défi de créer une oeuvre pour piano avec des éléments folkloriques dans la technique traditionnelle: il joint deux chants (Mehmétyo le et Tudora) en une sonorité pastel délicate et raffinée, en faisant un riccercare a six voix sur le thème de Tudora lors de son point culminant dans un climat serein:

Exemple 26

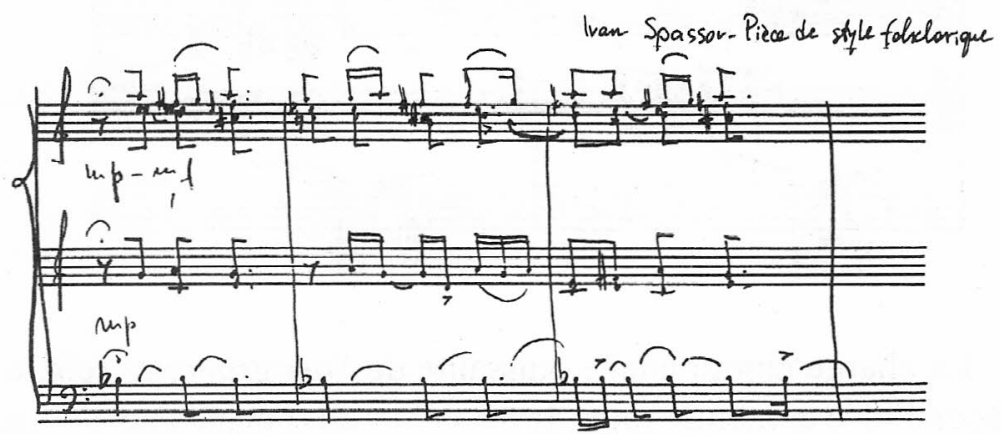

Guéorgui Mintchev fait partie du groupe de compositeurs les plus célèbres de par le monde. Son concerto pour piano (1978) a remporté de prestigieux prix internationaux, comme par exemple le prix d'art Medici en Florence, 1979. Il fut présenté au festival Automne de Varsovie, et en 1979 reçut l'éloge de la critique américaine comme étant une des meilleures oeuvres contemporaines 
de l'année. Les caractéristiques qualifiant le style de piano de Mintchev sont nombreuses, dont sa manière soulignée de transmettre ses idées musicales colorées dans une structure libre, la plénitude du son acquise par la recherche de chaque type d'extraction de sonorité et de dynamique, lesquelles se manifestent durant les improvisations (la captation impulsive de tout le diapason du piano), le jeu d'apaisement du son des tons d'harmonie allant plus loin jusqu'au grondement.

Ces particulatités du langage de composition de Mintchev correspondent à celle de son oeuvre intitulée Sonogrammes (5 réminiscences), écrite en 1980 à partir d'un matériel composé mais qui n'est pas inclu par l'auteur dans son concert pour piano. Il n'y a point de liaison dramaturgique entre elles, mais son idée très puissante et complète, se reflète dans ces pièces qui, de ce pas, se font accepter comme faisant partie d'une seule et même oeuvre divisée en cinq humeurs diverses.

\section{Exemple 27}

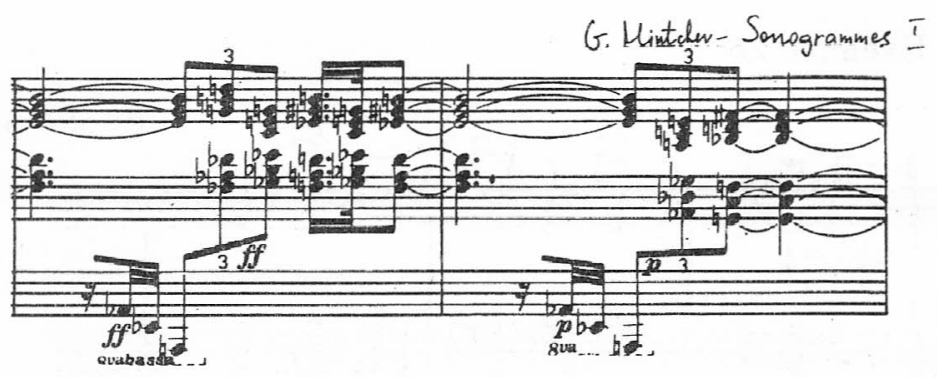

La charge émotionnelle puissante de Sonogrammes reflète à sa façon l'idée contemporaine de Mintchev du romantisme: l'auteur traduit les idées universelles pour tout le monde en la langue de son temps. Je crois que ceci est une explication de la grande influence de l'oeuvre sur le public.

Dimiter Hristov (1933) est l'auteur d'une grande oeuvre dédiée au piano: dix sonates (et encore deux à l'étape de projet), Toccata (1992), Chaconne (1984) et le cycle Imperméables ... après la 
pluie terrible (1994). Dans son essai dédié aux oeuvres pour piano de Dimiter Hristov, enregistrées sur CD par la compagnie Concord Concerto, Erick Salzmann dit: "L'on a recours au terme 'postmodernisme', trop souvent, d'après moi, parfois au sens vague. Mais si ceci signifiait un controle sévère et abstrait de la mélodie, l'harmonie et le rythme et la caractéristique timbrale du modernisme traditionnel se répandant sur les éléments d'inspiration de la musique populaire, la musique de D. Hristov s'intitulerait post-moderniste dans le plein sens du mot".

Les sonates sont toujours bipartites, d'une durée de dix minutes environ, composées de cycles de deux et trois; suite à sa première Sonate (1962), il créa un cycle global de trois sonates op. 25 (les Sonates n. ${ }^{\circ} 2$ op. 3 et n. ${ }^{\circ} 4$ de 1974); un cycle de cinq et six sonates op. 60 (1992-3) et un sycle de sept et huit, op. 70 (1994).

C'est une musique exceptionnellement classique pour piano, dans laquelle l'auteur utilise toutes les possibilités orchestrales du piano.

La personalité créatrice du compositeur, pianiste et architecte Dimitar Nenov (1901-1953) se dégage parmi les figures des compositeurs-innovateurs bulgares et il continue à susciter l'intérêt car son oeuvre est encore peu connue. La cause en sont les interdictions partielles de la part des cercles musicaux officiels et le manque de possibilité de propager son oeuvre après 1948. Dimiter Nenov est un pianiste de niveau européen qui se distingue parmi ses contemporains par la noblesse et l'envergure de ses intérpretations. Il fut un fervent adepte de la tradition de piano créée par Liszt, étant l'élève de Mm. ${ }^{e}$ von Kreutzer à Dresde, elle même élève de Liszt, et surtout d'Egon Petri. Nenov possède la capacité d'englober dans ses oeuvres le piano d'une façon exceptionnellement ample et pianistique, d'une façon unique en comparaison avec les autres compositeurs bulgares, sachant combiner avec une intuition raffinée le coloris musical. L'on pourrait le comparer avec Rachmaninov, étant un phénomène dans la vie culturelle bulgare. Bien qu'il vive dans la réalité de son temps, Nenov est porteur d'un sentiment purement moderne, plutôt impressioniste 
du point de vue de la sonorité qu'il découvre à sa propre manière quant aux thèmes folkloriques bulgares. Nous ne pouvons pas avancer la thèse que c'est un Bartók ou un Debussy bulgare. Mais cependant le refus du langage tonal chez Nenov est évident, surtout dans ses Deux Études op. 11. Il a recours à de nombreuses combinaisons modales-harmoniques bitonales, qui représentent une vibrante ligne de contact entre les accords majeurs et mineurs combinés en un seul point de retour obsédant vers un bref motif de départ, tout ceci recouvert d'un halo d'idées mystiques et symboliques.

\section{Exemple 28}

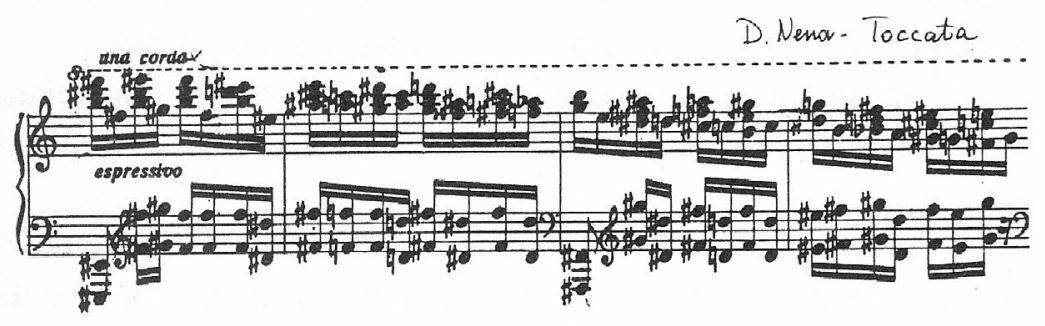

En ce qui concerne l'écriture, Nenov est l'adepte de la tradition post-Lisztienne d'une technique de piano d'envergure majeure: octaves, lignes de tièrces et de sixtes doublées, séquences de gamme d'accords complects. De sa propre volonté, il s'entoure exclusivement de la musique de Liszt, Beethoven, Scriabine, Chopin - musique qu'il interprète lui-même et sur laquelle il adapte sa technique de composition qui, dorénavant, sera l' objet d'une redécouverte. Nenov a laissé une oeuvre pour piano qui n'est pas grande en volume, car il n'a pas aimé transcrire les oeuvres qu'il improvise sur scène, et la part majeure de ses improvisations ne sont connues que de ses adeptes et ne sont restées que dans leurs souvenirs jusqu'à présent. Il a écrit un concert pour piano (1952), deux Études op. 11, un Thème avec variations, une Sonate, cinq Miniatures, six Préludes. 
Exemple 29

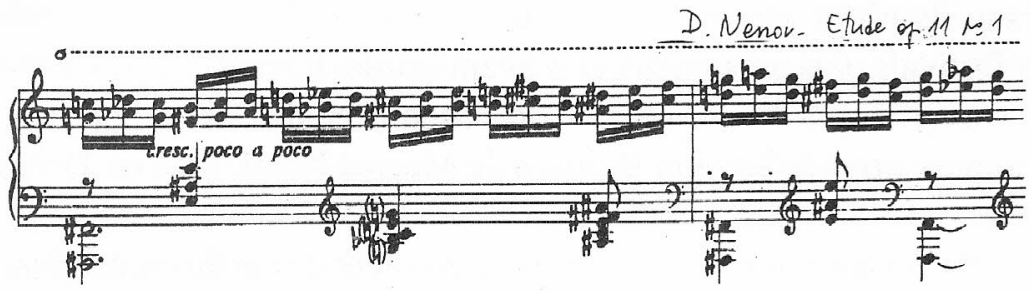

Les compositeurs nés après 1950 et demeurés dans le pays créent leurs oeuvres dans une atmosphère d'isolement dûe au manque d'un milieu quelconque qui puisse leur prêter aide économique et matérielle pour qu'ils puissent s'épanouir. L'époque du totalitarisme communiste, bien qu'avec des fins de propagande, disposait d'une structure musicale contraignante mais qui toutefois a prêté une certaine aide matérielle depuis les fonds d'état pour beaucoup de leurs créations et projets. Aujourd'hui, la concurrence libre et la sélection naturelle à la recherche d'un marché exercent leur influence de la façon la plus chaotique. La situation décide au moment même si un tel compositeur peut se faire jouer aujourd'hui ou non. Ce chaos dans lequel le corps de compositeurs est immergé dans tous les pays de l'Europe de l'Est pourrait se canaliser uniquement par voie d'évolution.

La cause en est le manque d'associations de compositeurs dans la vie sociale et culturelle de Bulgarie lorsque ceux-ci sont jeunes. L'indifférence et le manque d'intérêt envers la culture sont des phénomènes à l'échelle universelle et représentent un problème global. Parfois la manque d'intérêt et le silence de la part du public sont encore plus durs à supporter qu'une critique négative.

Dans une situation telle que celle-ci, cependant, quelques compositeurs réussirent à trouver leur chemin créateur par la voie d'un vrai essor culturel. Leurs idées de base sont tout à fait détachées de la réalité sociale du pays. Ils vivent dans un isolement intérieur mais avec le droit de liberté sur leur activité créatrice, "établi après 
1990 en tant qu'acte officiel de la vie spirituelle de Bulgarie". Grâce à la mondialisation de l'information, ils ne son plus isolés, même lorsqu'ils n'ont pas les moyens de faire jouer leurs oeuvres dans des festivals plus prestigieux et d'avant-garde. Il reste une possibilité de s'exprimer à l'extérieur et de rencontrer des avant-gardistes étrangers pour la Section Bulgare de Musica Nova, créé en 1990 à Sofia.

Parmi les jeunes auteurs qui s'expriment d'une façon originale à travers le langage du piano, nous noterons Guéorgui Arnaoudov (1957). C'est l'auteur des pièces Svarog Rituel I, Incarnation dans la lumière (Rituel II), lesquels expriment de façon très brillante son langage, défini par certains critiques musicaux comme modalminimaliste. Toutefois un motif, ou un seul intervalle (le plus souvent une seconde ou une quinte) deviènnent porteurs d'une lente accumulation d'immense énergie et de puissance à la façon d'une avalanche qui atteint un sommet et qui, plus tard, se décompose en allant vers le néant. Le plus souvent le sommet lui même contient des intervalles ou des accords identiques obstinés qui se repètent souvent et longuement et qui paraissent épuiser jusqu'à la limite toute autre possibilité de developpement postérieur.

\section{Exemple 30}

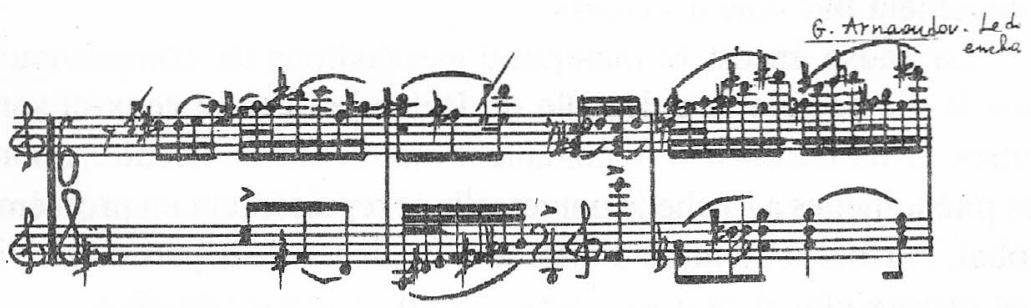

Dans sa trilogie intitulée Un pan de ciel au milieu du silence, écrite sur les trois tableaux du même nom de René Magritte ( $L$ 'empire des lumières, Le domaine enchanté et La voix des airs) /1991-1995/, Arnaoudov démontre son crédo superexpressif et 
romantique de la musique, énoncé en des structures modales déployées de façon minimale.

L'auteur lui-même déclare avoir essayé de créer diverses sonorités cristaliines qui résonnent à l'unisson: "Mon but était de diriger l'énergie des sons vers la ritualité, vers le retour de l'Ego de l'homme à la nature et à Dieu".

L'oeuvre pianistique d'Arnaoudov a été enregistré sur CD par la compagnie américaine Concord Concerto (1996), interprétée par l'auteur du présent écrit.

Nous pouvons citer d'autres jeunes compositeurs bulgares intéressants, auteurs d'oeuvres pour piano, comme Dragomir Yossifov (1964): 18 Bagatelles (1996); et Vesselin Karaatanasov (1966): Perplexities (1996).

Parmi les jeunes qui vivent à l'étranger depuis 1989, nous pouvons faire mention des noms suivants: Bojidar Spassov (1949), vivant en Bohum, Allemagne; Mikhail Goléminov (1956), vivant à Viènne, Autriche; Alexandre Kandov (1949), Madrid, Espagne; et le pianiste émérite Boyan Vodenitcharov (1960), qui vit à Bruxelles, en Belgique. Ce dernier a réalisé l'enregistrement sur disque CD de plusieurs oeuvres pour piano de ces derniers auteurs en 1994. Ces oeuvres disposent d'une image propre et d'un niveau professionnel de très haute catégorie par lesquels elles arrivent à capter de façon très particulière l'époque contemporaine. Dans ses Cascades (1991) Bojidar Spassov réussit à réaliser un apport de maître à la tradition post-Darmstadt en y ajoutant une note slave. Mikhail Goléminov, dans son Klavierstuck I (1992), renforce de façon exceptionnelle son orientation philosophique comparée à ses opus créés en Bulgarie, étant plus jeune, et, à la différence de Bojidar Spassov, se maintient beaucoup plus près des traditions purement européennes du post-sérialisme.

Chez Mikhail Goléminov nous ne découvrons aucune trace d'idées qui lui sont propres, provenant de la façon slave de penser.

Nous pourrions nommer Alexandre Kandov post-moderniste (né en 1949), et ceci en raison de la substance synthétique de sa musique où les frontières entre style littéraire, art, image et son 
s'estompent. Il exprime l'esthétique du rock, punck, underground ou new wave. Il crée son oeuvre pour piano Noctuelles (1993) en tant que partie d'un grand cycle de papillons: Papillons éxotiques, Papillons africains et Papillons de perle.

Boyan Vodenitcharov créa son Praeludium II en 1994. Son orientation vers les interprétations de jazz l'a mené de façon tout à fait naturelle vers la symbiose entre l'improvisation et les structures diatoniques classiques simplifiées, lesquelles créent plutôt un sentiment de volume que de mouvement, suivant les dires de l'auteur.

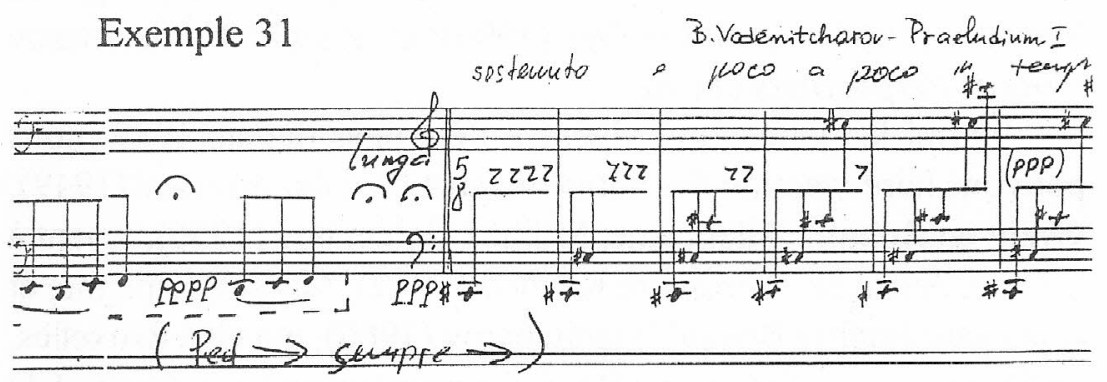

\section{NOTES}

1. La réaction continue contre les compositeurs Konstantine Iliev et Lazare Nikolov durant la période des années cinquante aux années quatre-vingt n'a pas été necéssairement guidée par des motifs politiques: tout simplément notre société musicale n'était pas prête à admettre des manifestations créatrices aussi avancées. Ceci est une réaction provenant de l'évolution de la liberté de l'homme en Bulgarie. Il est bien vrai que ce fut pour la première fois, en Janvier de 1990, durant le 14-ème Congrès du P.C.B., que son Secrétaire Général, Pétar Mladenov, declara que la Bulgarie donne libre droit à toute intention créatrice et chaque créateur obtient droit d'existence légale.

2. Koukeres: Costumes et masques paiens servant à chasser les mauvais esprits durant des cérémonies populaires annuelles.

Angela Tosheva, nascida e residente na Bulgária, é professora e pianista, intérprete, entre outros autores ocidentais, dos compositores contemporâneos búlgaros. 